\title{
IDENTITIES OF THE NATURAL REPRESENTATION OF THE INFINITELY BASED SEMIGROUP
}

\author{
LEONID AL'SHANSKII AND ALEXANDER KUSHKULEY
}

(Communicated by Andreas R. Blass)

\begin{abstract}
An equational theory of a very small semigroup may fail to be finitely presented. A well-known example of such a semigroup was studied in detail by Peter Perkins some twenty years ago. We prove that the natural representation of his semigroup has a finite basis of identical relations and discuss this fact in a general context of universal algebra.
\end{abstract}

\section{INTRODUCTION}

Let $k$ be a field and let $e_{i j}, 1 \leq i, j \leq 2$, be the matrix units of the algebra $M_{2}(k)$ of $2 \times 2$ matrices over $k$. These four matrix units together with the zero matrix $O$ and the identity matrix $E$ form the semigroup $\Pi$ that does not possess a finite base of identities (see [1]). Nevertheless, in this paper we prove the following

Theorem 1. The natural representation id: $\Pi \rightarrow M_{2}(k)$ of the semigroup $\Pi$ is finitely based. A particular basis of identities of this representation consists of the identities (1)-(7).

The semigroup $\Pi$ acts by (left) multiplications on $M_{2}(k)$. The regular representation of $\Pi$ in its semigroup algebra splits up into the direct sum of this fourdimensional representation and two one-dimensional representations. Hence we have

Corollary 1. Any representation of the semigroup $\Pi$ is finitely based.

\section{BASIC DEFINITIONS}

Identities of representations of semigroups can be naturally defined as follows (see [2]). Let $F \equiv F(X), X=\left\{x_{1}, x_{2}, \ldots, y_{1}, y_{2}, \ldots, z_{1}, z_{2}, \ldots\right\}$, be the free semigroup with the countable set of free generators $X$, and let $k F$ be its semigroup algebra. Take a representation of a semigroup $S$ by the linear transformations of a vector space $V$. A polynomial $P \equiv p\left(x_{1}, \ldots, x_{t}\right) \in k F$ is said to be an identity of such $r: S \rightarrow$ End $V$ (of the pair (End $V, S)$ ) if $p\left(r\left(s_{1}\right), \ldots, r\left(s_{t}\right)\right)=0$ for all $s_{1}, \ldots, s_{t} \in S$.

Received by the editors June 14, 1989 and, in revised form, October 9, 1991.

1991 Mathematics Subject Classification. Primary 03C05, 03C13, 03C60, 20M07, 20M20. 
Example. If an identity $f=g$ holds in a semigroup then the identity $f-g$ holds in any of its representations (for faithful representations the converse is also true). More generally, if the universal disjunctive formula (pseudoidentity) $w \equiv f_{1}=g_{1} \vee \cdots \vee f_{m}=g_{m}$ holds in a semigroup $S\left(f_{i}, g_{i} \in F, i=1, \ldots, m\right)$ then, clearly, the identities

$$
u(w)=\left(f_{1}-g_{1}\right) x_{1}^{\nu_{1}} \cdots x_{m-1}^{\nu_{m-1}}\left(f_{m}-g_{m}\right), \quad \nu_{1}, \ldots, \nu_{m-1} \in\{0,1\},
$$

hold in the regular representation $(k S, S)$.

In what follows the word "identity" will mean an identity of a representation. Let polynomials $p_{1}, \ldots, p_{n}$ be identities of (End $\left.V, S\right)$. Then for any endomorphisms $\varepsilon_{i}: F \rightarrow F$ and any $a_{i}, b_{i} \in k F(i=1, \ldots, n)$ the polynomial

$$
a_{1} \varepsilon_{1}\left(p_{1}\right) b_{1}+\cdots+a_{n} \varepsilon_{n}\left(p_{n}\right) b_{n}
$$

is also an identity of (End $V, S$ ). Let $I \subset k F$ be the set of all the identities of the pair (End $V, S$ ). Clearly, $I$ is an ideal in $k F$. Moreover, it is a verbal ideal, which means that all the expressions (2.1) with $p_{1}, \ldots, p_{n} \in I$ also belong to $I$. A set $B \subset I$ is called a basis of the identities of (End $V, S)$ (a basis of $I$ ) if any $q \in I$ can be written in the form (2.1) with $p_{1}, \ldots, p_{n} \in B$. The problem is to identify the situations in which such a $B$ can be finite. In this latter case, a representation (or the verbal ideal of its identities) is called finitely based.

\section{Proof of Theorem 1}

Consider a monomial

$$
M=p_{1}\left(a_{1}^{2}-a_{1}\right) p_{2}\left(a_{2}^{2}-a_{2}\right) \cdots p_{r}\left(a_{r}^{2}-a_{r}\right) p_{r+1} \in k F,
$$

where $p_{1}, \ldots, p_{r+1} \in F$ are possibly empty products of the squares of free variables, $a_{1}, \ldots, a_{r} \in X \quad(r \geq 0)$. We will use the following notations: $A(M):=\left\{a_{1}, \ldots, a_{r}\right\}, Y(M):=\left\{x \in X, x^{2}\right.$ occurs in at least one of the monomials $\left.p_{1}, \ldots, p_{r+1}\right\} ; l(M):=r ; o(M):=\{x \in X, x$ equals some of the $a_{i}$ with $i$ odd $\} ; e(M):=\left\{x \in X, x\right.$ equals some of the $a_{i}$ with $i$ even $\}$. For $x \in A(M)$ let $n_{x}(M):=\left\{\right.$ the number of occurrences of $x^{2}-x$ in $\left.M\right\}$. For an arbitrary $h \in k F$ let $X(h):=\{x \in X, x$ occurs in $h\}$. Clearly, $X(M)=A(M) \cup Y(M)$ and $A(M)=o(M) \cup e(M)$. The homomorphism $k F \rightarrow M_{2}(k)$ induced by a map $\sigma: X \rightarrow \Pi$ will be denoted by the same letter. Set $U:=\left\{E, e_{11}, e_{22}\right\}, N:=\left\{e_{12}, e_{21}\right\}, U_{0}:=U \cup 0$, and $N_{0}:=N \cup 0$.

Lemma 1. A map $\sigma: X \rightarrow \Pi$ such that $\sigma(M) \neq 0$ exists iff the following conditions are satisfied:

(i) $\sigma(A(M)) \subset N$ and $\sigma(Y(M)) \subset U$; in particular, $A(M) \cap Y(M)=\varnothing$;

(ii) $\sigma(o(M)) \cap \sigma(e(M))=\varnothing$, i.e., there are only two possibilities: either $\sigma(o(M))=\left\{e_{12}\right\}, \sigma(e(M))=\left\{e_{21}\right\}$, and $\sigma(M) \in k e_{12} \oplus k e_{11}$ or $\sigma(o(M))=$ $e_{12}, \sigma(e(M))=e_{12}$, and $\sigma(M) \in k e_{21} \oplus k e_{22}$; in particular, $o(M) \cap e(M)=\varnothing$.

Lemma 2. $M$ is an identity of $\left(M_{2}(k), \Pi\right)$ iff $A(M) \cap Y(M) \neq \varnothing$ or $o(M) \cap$ $e(M) \neq \varnothing$.

The proofs of these lemmas are straightforward and rely on the following trivial observation.

Remark 1. If $s \in \Pi$ then either $s^{2}-s=0 \quad\left(s \in U_{0}\right)$ or $s^{2}=0 \quad\left(s \in N_{0}\right)$. 
Lemma 3. The pair $\left(M_{2}(k), \Pi\right)$ satisfies the following identities:

$$
\begin{gathered}
x^{3}-x^{2} \\
x^{2} y^{2}\left(x^{2}-x\right), \quad\left(x^{2}-x\right) y^{2} x^{2} ; \\
\left(x^{2}-x\right) y^{2}\left(x^{2}-x\right) ; \\
\left(x_{1}^{2}-x_{1}\right) y^{2 \nu}\left(x_{2}^{2}-x_{2}\right)\left(x_{3}^{2}-x_{3}\right)-\left(x_{3}^{2}-x_{3}\right) y^{2 \nu}\left(x_{2}^{2}-x_{2}\right)\left(x_{1}^{2}-x_{1}\right) ; \\
\left(x_{1}^{2}-x_{1}\right) y^{2 \nu}\left(x_{2}^{2}-x_{2}\right) z^{2}-z^{2}\left(x_{1}^{2}-x_{1}\right) y^{2 \nu}\left(x_{2}^{2}-x_{2}\right) ; \\
\left(x^{2}-x\right) z^{2 \nu}\left(y^{2}-y\right)\left(x^{2}-x\right)\left(y^{2}-y\right)=\left(x^{2}-x\right) z^{2 \nu}\left(y^{2}-y\right) ; \\
x^{2} y^{2}=(x y x)^{2}=y^{2} x^{2}
\end{gathered}
$$

(here $\nu \in\{0,1\}$ so that each of the expressions (4)-(6) represents a pair of identities).

All these identities can be easily verified with the use of Lemmas 1, 2 .

Remark 2. It follows from (7) that the identities (2)-(6) remain valid if one substitutes any products of squares of free variables instead of $y^{2}$ and $z^{2}$.

Remark 3. The identities $\left(x^{2}-x\right)^{m}, m \geq 2$, and $x^{i}-x^{j}, i, j \geq 2$, follow from (1).

Denote by $V$ the verbal ideal generated by the identities (1)-(7).

Remark 4. Let an endomorphism $\varphi: F \rightarrow F$ be such that $\varphi \mid Y(M) \backslash A(M)=$ id, $\varphi(o(M))=o(M), \varphi(e(M))=e(M)$. Then the identity (4) shows that $\varphi(M) \equiv M(\bmod V)$.

Take an identity $f=f\left(x_{1}, \ldots, x_{t}\right) \in k F$ of $\left(M_{2}(k), \Pi\right)$ and write $x_{i}=$ $x_{i}^{2}-\left(x_{i}^{2}-x_{i}\right), i=1,2, \ldots, t$, in order to get

$$
f=\sum_{i=1}^{n} \alpha_{i} M_{i},
$$

where $\alpha_{i} \in k$ and $M_{i}$ are monomials of the form (3.1). Assume that (3.2) is minimal, i.e., $\sum_{i \in I} \alpha_{i} M_{i}$ is not an identity of $\left(M_{2}(k), \Pi\right)$ for any proper subset $I \subset\{1, \ldots, n\}$. To prove the theorem it is sufficient to show that $f \in V$. If $n=1$ then this follows from Lemma 2 and Remarks 2, 3, and 4. So we assume that $n>1$.

Lemma 4. For any $i, j \in\{1,2, \ldots, n\}$ the following conditions hold:

(i) $X\left(M_{i}\right)=X\left(M_{j}\right)$;

(ii) $A\left(M_{i}\right) \cap Y\left(M_{i}\right)=\varnothing, o\left(M_{i}\right) \cap e\left(M_{i}\right)=\varnothing$;

(iii) $A\left(M_{i}\right)=A\left(M_{j}\right), Y\left(M_{i}\right)=Y\left(M_{j}\right)$;

(iv) $o\left(M_{i}\right)=o\left(M_{j}\right), e\left(M_{i}\right)=e\left(M_{j}\right)$;

(v) $l\left(M_{i}\right)=l\left(M_{j}\right)(\bmod 2)$.

Proof. Condition (i) is obvious-it means that $f$ is "blended" in the sense of [5, p. 15].

Condition (ii) follows from the minimality assumption and Lemma 2.

To prove (iii) suppose that $x \in A\left(M_{i}\right) \backslash A\left(M_{j}\right)$ and let

$$
f^{\prime}=\sum_{x \in A\left(M_{t}\right)} \alpha_{t} M_{t} \text { and } f^{\prime \prime}=\sum_{x \notin A\left(M_{t}\right)} \alpha_{t} M_{t} .
$$


By Lemma 1, $\sigma(x) \in N$ for any $\sigma: X \rightarrow \Pi$ such that $\sigma\left(f^{\prime}\right) \neq 0$, and $\sigma(x) \in U$ for any $\sigma: X \rightarrow \Pi$ such that $\sigma\left(f^{\prime \prime}\right) \neq 0$. Hence $\sigma\left(f^{\prime}\right)=0$ or $\sigma\left(f^{\prime \prime}\right)=0$ for every $\sigma: X \rightarrow \Pi$. This contradicts the minimality assumption (note that $\left.f=f^{\prime}+f^{\prime \prime}\right)$. Further, if $o\left(M_{i}\right) \neq o\left(M_{j}\right)$ then again write

$$
f=f^{\prime}+f^{\prime \prime}=\sum_{o\left(M_{t}\right)=o\left(M_{i}\right)} \alpha_{t} M_{t}+\sum_{o\left(M_{t}\right) \neq o\left(M_{i}\right)} \alpha_{t} M_{t}
$$

and suppose that $\sigma\left(f^{\prime}\right) \neq 0$ for some $\sigma: X \rightarrow \Pi$. Using (iii) and Lemma 1, one easily verifies that either $\sigma\left(o\left(M_{i}\right)\right)=e_{12}, \sigma\left(f^{\prime}\right) \in k e_{11} \oplus k e_{12}, \sigma\left(f^{\prime \prime}\right) \in$ $k e_{21} \oplus k e_{22}$ or $\sigma\left(o\left(M_{i}\right)\right)=e_{21}, \sigma\left(f^{\prime}\right) \in k e_{21} \oplus k e_{22}, \sigma\left(f^{\prime \prime}\right) \in k e_{11} \oplus k e_{12}$. Both cases contradict $\sigma(f)=0$. This proves (iv). The proof of (v) is similar.

In view of Lemma 4 we can use the notation $X(f), A(f)$, etc.

Lemma 5. There exists a polynomial $f^{\prime}=\sum_{i=1}^{n} \alpha_{i} M_{i}^{\prime}$ such that $f-f^{\prime} \in V, f^{\prime}$ satisfies all the conditions of Lemma 4 , and, in addition, $n_{x}\left(M_{i}^{\prime}\right)=n_{x}\left(M_{j}^{\prime}\right)$ for all $x \in A\left(f^{\prime}\right), i, j \in\{1,2, \ldots, n\}$.

Proof. Let $m_{y}=\max _{1 \leq i \leq n}\left\{n_{y}\left(M_{i}\right)\right\}, y \in e(F)$. Fix a variable $x \in o(f)$. Set

$$
M_{i}^{\prime \prime}= \begin{cases}M_{i} \prod_{v \in e(f)}\left[\left(x^{2}-x\right)\left(v^{2}-v\right)\right]^{m_{v}-n_{v}\left(M_{i}\right)} & \text { if } l(f) \text { is even, } \\ M_{i} \prod_{v \in e(f)}\left[\left(v^{2}-v\right)\left(x^{2}-x\right]^{m_{v}-n_{v}\left(M_{i}\right)}\right. & \text { otherwise. }\end{cases}
$$

If $y \in e(f)$ then, clearly, $n_{y}\left(M_{i}^{\prime \prime}\right)=m_{y}, i=1,2, \ldots, n$. Let $m_{v}^{\prime \prime}=$ $\max _{1 \leq i \leq n}\left\{n_{v}\left(M_{i}^{\prime \prime}\right)\right\}, v \in o(f)$. Fix a variable $y \in e(f)$ and again set

$$
M_{i}^{\prime}= \begin{cases}\prod_{v \in o(f)}\left[\left(y^{2}-y\right)\left(v^{2}-v\right)\right]_{v}^{m_{v}^{\prime \prime}-n_{v}}\left(M_{i}^{\prime \prime}\right) & \text { if } l(f) \text { is odd } \\ \prod_{v \in o(f)}\left[\left(v^{2}-v\right)\left(y^{2}-y\right)\right]^{m_{v}^{\prime \prime}-n_{v}\left(M_{i}^{\prime \prime}\right)} & \text { if } l(f) \text { is even. }\end{cases}
$$

If $x \in o(f)$ then $n_{x}\left(M_{i}^{\prime}\right)=m_{x}, i=1, \ldots, n$, as above. On the other hand, if $x \in e(f) \backslash y$ then $n_{x}\left(M_{i}^{\prime}\right)=n_{x}\left(M_{i}^{\prime \prime}\right)=m_{x}, i=1,2, \ldots, n$. Finally,

$$
n_{y}\left(\boldsymbol{M}_{i}^{\prime}\right)=m_{y}+\sum_{v \in o(f)}\left(m_{v}^{\prime \prime}-n_{v}\left(\boldsymbol{M}_{i}^{\prime \prime}\right)\right.
$$

but

$$
\sum_{v \in o(f)} n_{v}\left(M_{i}^{\prime \prime}\right)=\sum_{u \in e(f)} n_{u}\left(M_{i}^{\prime \prime}\right)+\nu=\sum_{u \in e(f)} m_{u}+\nu
$$

where $\nu=0$ if $l(f)$ is even and $\nu=1$ otherwise. So we have $n_{x}\left(M_{i}^{\prime}\right)=$ $n_{x}\left(M_{j}^{\prime}\right)$ for all $i, j=1,2, \ldots, n$. Moreover, it follows from (6) and Remark 4 that $M_{i}^{\prime} \equiv M_{i}(\bmod V)$. Note also that $X\left(M_{i}^{\prime}\right)=X(f), A\left(M_{i}^{\prime}\right)=A(f)$, etc.

Lemma 6. Modulo the ideal $V$, the identity $f$ equals

$$
\sum_{i, j} \alpha_{i j} M_{i j} \equiv \sum_{i, j} \alpha_{i j} p_{i}\left(a_{1}^{2}-a_{1}\right) q_{j}\left(a_{2}^{2}-a_{2}\right) \cdots\left(a_{t}^{2}-a_{t}\right),
$$

where $\alpha_{i j} \in k, p_{i}, q_{j}$ are the products of squares of the variables belonging to $Y(f), a_{r} \in o(f)$ if $r$ is odd, and $a_{r} \in e(f)$ otherwise.

Proof. Apply (4), (5), and Remark 4 to an identity $f^{\prime}$ that satisfies the conditions of Lemma 5.

Obviously $X\left(p_{i}\right) \cup X\left(q_{j}\right)=Y(f)$ for all $i, j$. We may suppose also that $\left\langle X\left(p_{i}\right), X\left(q_{j}\right)\right\rangle=\left\langle X\left(p_{i}^{\prime}\right), X\left(q_{j}^{\prime}\right)\right\rangle$ iff $i=i^{\prime}, j=j^{\prime}$. To conclude the proof of 
the theorem, we will show by induction on $\operatorname{Card}\left(X\left(p_{i}\right) \cap X\left(q_{j}\right)\right)$ that all $\alpha_{i j}$ in (4.3) are zeros. Let

$$
\begin{gathered}
\sigma_{i j}\left(a_{r}\right)= \begin{cases}e_{12} & \text { if } r \equiv 1 \quad(\bmod 2), \\
e_{21} & \text { if } r \equiv 0(\bmod 2) ;\end{cases} \\
\sigma_{i j}(y)= \begin{cases}E & \text { if } y \in X\left(p_{i}\right) \cap X\left(q_{j}\right), \\
e_{11} & \text { if } y \in X\left(p_{i}\right) \backslash X\left(q_{j}\right), \\
e_{22} & \text { if } y \in X\left(q_{j}\right) \backslash X\left(p_{i}\right) .\end{cases}
\end{gathered}
$$

Note that this definition is correct because of Lemmas 4 and 5 .

Suppose that $\alpha_{r s}=0$ if $\operatorname{Card}\left(X\left(p_{r}\right) \cap X\left(q_{s}\right)\right)<\operatorname{Card}\left(X\left(p_{i}\right) \cap X\left(q_{j}\right)\right)$. Clearly $\sigma_{i j}\left(M_{i j}\right)=e_{12}$ if $t$ is odd and $\sigma_{i j}\left(M_{i j}\right)=e_{11}$ otherwise. On the other hand, if $\sigma_{i j}\left(M_{r s}\right) \neq 0$ then $X\left(p_{r}\right) \subset X\left(p_{i}\right), X\left(q_{s}\right) \subset X\left(q_{j}\right)$. Therefore $X\left(p_{r}\right) \cap X\left(q_{s}\right) \subset$ $X\left(p_{i}\right) \cap X\left(q_{j}\right)$ and by induction $X\left(p_{r}\right) \cap X\left(q_{s}\right)=X\left(p_{i}\right) \cap X\left(q_{j}\right)$. All this means that $X\left(p_{r}\right)=X\left(p_{i}\right), X\left(q_{s}\right)=X\left(q_{j}\right)$, and $\langle r, s\rangle=\langle i, j\rangle$. But $\sigma_{i j}(f)=0$, and hence $\alpha_{i j}=0$.

\section{CONCLUDING REMARKS}

4.1. Theorem 1 implies in particular that the infinite set of identities of the semigroup $\Pi$ that was described in [1] can be derived from the identities (1)-(7) (in the sense of $\S 2$ ). It is not very difficult to show this directly.

4.2. Consider the semigroup $\Pi^{\prime}=\Pi \backslash E$. The natural representation of this semigroup satisfies the identity

$$
y^{2}\left(x^{2}-x\right) y^{2}
$$

Minor changes in the above proof of Theorem 1 (note that $X\left(p_{i}\right) \cap X\left(q_{j}\right)=\varnothing$ because of $(8)$ ) yield

Corollary 2. The identities (1)-(8) constitute a basis of identities of the natural representation of $\Pi^{\prime}$.

Corollary 3. All representations of $\Pi^{\prime}$ are finitely based. A finite basis of identities of the semigroup $\Pi^{\prime}$ was written down in [6].

4.3. Theorem 1 provides an illustration for a more general situation that we will briefly discuss. Let $A$ be an algebraic system of signature $\Omega$ ( $\Omega$-algebra). A representation of $A$ is a map $r: A \rightarrow B$ into a $\Sigma$-algebra $B$ such that for any $w \in \Sigma$

$$
r\left(w\left(a_{1}, \ldots, a_{t}\right)\right)=\sigma_{w}\left(r\left(a_{1}\right), \ldots, r\left(a_{t}\right)\right),
$$

where $\sigma_{w} \in \Sigma$ and $a_{1}, \ldots, a_{t} \in A$. Identities of representations can be defined in this general setting (cf. [3]).

Conjecture. Any algebraic system possesses a faithful finitely based representation into an algebra of (reasonably) extended signature.

Example. Let $K$ be an algebraically closed field. Call a $\Sigma$-algebra polynomial if there exists an injective map $\varphi: A \rightarrow K^{n}$ such that for any $w \in \Omega$

$$
\lambda_{s}\left(\varphi\left(w\left(a_{1}, \ldots, a_{t}\right)\right)\right)=P_{s}^{w}\left(\lambda_{1}\left(\varphi a_{1}\right), \ldots, \lambda_{n}\left(\varphi a_{1}\right), \ldots, \lambda_{n}\left(\varphi a_{t}\right)\right),
$$


where $P_{s}^{w}$ is a polynomial in $n t$ variables, $\lambda_{s}: K^{n} \rightarrow K$ is the $s$ th coordinate function, $s=1,2, \ldots, n$, and $a_{1}, \ldots, a_{t} \in A$. Fix a basis $e_{1}, \ldots, e_{n} \in K^{n}$ and consider the two-carrier algebra $\left(K^{n}, K\right)$, which apart from the usual vector space operations, includes the following:

$e_{1}, \ldots, e_{n} \in K^{n}$-nullary operations;

$P^{w}:\left(K^{n}\right)^{t} \rightarrow K^{n}, w \in \Omega$ - the operations defined by the right-hand sides of the relation (4.2) (i.e., the $s$ th coordinate of $P^{w}(x)$ equals $P_{s}^{w}$ (coordinates of $x))$;

$\lambda_{s}: K^{n} \rightarrow K, s=1,2, \ldots, n$-the coordinate functions.

The map $\varphi: A \rightarrow K^{n}$ satisfies the conditions (4.1) with $\sigma_{w}=p^{w}$. Hence one has the representation (triple) $\left(A, K^{n}, K\right)$.

Theorem 2. The triple $\left(A, K^{n}, K\right)$ is finitely based.

We mention also some of the natural open questions that arise in connection with Theorem 2.

(a) What is the 'minimal' extension of a signature that ensures a finite basis of identities?

(b) What remains of Theorem 2 when $K^{n}$ is replaced by an infinite-dimensional space? tion?

(c) Does every semigroup (group) possess a finitely based linear representa-

4.4. It is possible that any linear representation of a finite semigroup is finitely based. We will state here without proof one partial result in this vein. The result shows that the example from $\S 2$ is a rather general one.

Theorem 3. Let $B$ be a basis of pseudoidentities of the semigroup $S$. Then some power of any identity of the representation $(k S, S)$ belongs to the verbal ideal generated by the set $\{u(b), b \in B\}$.

Corollary. Let $S$ be a finite semigroup. Then the verbal ideal I of identities of its regular representation contains a finitely based (verbal) ideal $I_{0}$ such that $I / I_{0}$ is a nil-algebra.

Proof. It is well known (see, e.g., [4]) that the positive universal theory of a finite algebraic system is finitely based.

The question of whether the regular representation of the semigroup $\Pi$ is finitely based was asked by Plotkin in the late seventies. A connection between positive universal formulas and identities of group representations was studied in a joint paper of Plotkin and Kushkuley (unpublished).

\section{REFERENCES}

1. Peter Perkins, Bases for equational theories of semigroups, J. Algebra 11 (1968), 298-314.

2. B. I. Plotkin, Varietes of representations of groups, Uspekhi Mat. Nauk. 32 (1977), 3-68. (Russian)

3. Ju. P. Razmyslov, Varieties of representations of finite dimensional algebras in prime algebras, Vestnik MGU 6 (1983), 31-37. (Russian)

4. P. M. Cohn, Universal algebra, Harper and Row, New York, 1965. 
5. Nathan Jacobson, PI-algebras, Lecture Notes in Math., vol. 441, Springer-Verlag, Berlin and New York, 1975.

6. A. N. Trahtman, A base of identities of the five-element semigroup of Brandt, Research in Modern Algebra, Sverdlovsk Univ., Sverdlovsk, 1987, pp. 147-149. (Russian)

Department of Applied Mathematics, Riga Polytechnic Institute, Latvia

Mathsoft, Inc., 201 Broadway, Cambridge, Massachusetts 02139

E-mail address: alex@mathsoft.com 